\title{
Polyelectrolyte Adsorption on Charged Substrate
}

\author{
Chi-Ho Cheng* and Pik-Yin Lai* \\ ${ }^{*}$ Department of Physics and Center for Complex Systems, National Central University, Taiwan, Republic of
} China.

\begin{abstract}
The behavior of a polyelectrolyte adsorbed on a charged substrate of high-dielectric constant is studied by both Monte-Carlo simulation and analytical methods. It is found that in a low enough ionic strength medium, the adsorption transition is first-order where the substrate surface charge still keeps repulsive. The monomer density at the adsorbed surface is identified as the order parameter. It follows a linear relation with substrate surface charge density because of the electrostatic boundary condition at the charged surface. During the transition, the adsorption layer thickness remains finite. A new scaling law for the layer thickness is derived and verified by simulation.
\end{abstract}

\section{INTRODUCTION}

The problem of polymer adsorption on an attractive surface has drawn considerable interest due to its relation to surface effects in critical phenomena and practical importance in technology and biology. It is well established that the adsorption transition is continuous if its attraction on the surface is short-ranged [1]. On the other hand, long-ranged electrostatic interactions in polyelectrolyte systems pose many challenging theoretical problems. Recently the macroion adsorption on an electrostatically attractive interface [2, 3] and the associated charge inversion phenomena of adsorbed polyelectrolytes [4, 5] acquire lots of attention.

Previous analytical approaches impose the continuity of monomer density across the charged surface, the surface monomer density is then set to zero [6, 7]. The polyelectrolyte adsorption problem becomes the competition between the electrostatic force from charged surface and the configurational entropy of the polyelectrolyte itself. The transition has been shown to be continuous. However, we found that the above treatment is not quite adequate since the electrostatic boundary condition at the charged surface is not faithfully respected. Furthermore, the electrostatic boundary condition may induce different physics in the presence of a substrate under the charged surface. In this paper, we study the adsorption of a polyelectrolyte on a high-dielectric substrate at low ionic strength (e.g. aqueous solution with a metal substrate) in which image charge interaction is attractive. The adsorption transition occurs where the surface charges are repulsive instead of the attractive case that were usually studied. The problem is tackled by Monte-Carlo simulations and analytical methods taking full account of appropriate boundary conditions. It is found that the order of adsorption transition, the physical mechanism, and the scaling behavior are all different from those of the attractive surfaces.

\section{MODEL}

A polyelectrolyte carrying positive charges is immersed in a medium $(z>0)$ of dielectric constant $\varepsilon$. At $z=0$ there is an impenetrable surface of uniformly surface charge density $\sigma$. Below that $(z<0)$, it is a substrate of dielectric constant $\varepsilon^{\prime}$. Denote the charge on a polymer segment $d s$ by $q_{0} d s$, the Hamiltonian is written as

$$
\begin{aligned}
\mathscr{H}= & \frac{3 k_{\mathrm{B}} T}{2 l_{0}^{2}} \int_{0}^{N} d s\left(\frac{\partial \vec{r}(s)}{\partial s}\right)^{2}+\frac{1}{2} \int_{0}^{N} d s \int_{0}^{N} d s^{\prime}\{ \\
& \left.\Gamma \frac{\mathrm{e}^{-\kappa\left|\vec{r}(s)-\vec{r}\left(s^{\prime}\right)\right|}}{\left|\vec{r}(s)-\vec{r}\left(s^{\prime}\right)\right|}-\Gamma^{\prime}\left(2-\delta_{s, s^{\prime}}\right) \frac{\mathrm{e}^{-\kappa\left|\vec{r}(s)-\vec{r}^{\prime}\left(s^{\prime}\right)\right|}}{\left|\vec{r}(s)-\vec{r}^{\prime}\left(s^{\prime}\right)\right|}\right\} \\
& +h \int_{0}^{N} d s \kappa^{-1} \mathrm{e}^{-\kappa \vec{r}(s) \cdot \hat{z}} \\
& +\omega \int_{0}^{N} d s \int_{0}^{N} d s^{\prime} \delta\left(\vec{r}(s)-\vec{r}\left(s^{\prime}\right)\right)
\end{aligned}
$$

where $s$ is the variable to parametrize the chain, $l_{0}$ the bare persistence length, and $\kappa^{-1}$ the Debye screening length. $\vec{r}(s)=(x(s), y(s), z(s))$, $\vec{r}^{\prime}\left(s^{\prime}\right)=\left(x\left(s^{\prime}\right), y\left(s^{\prime}\right),-z\left(s^{\prime}\right)\right)$ are the positions of the monomers and their electrostatic images, respectively. $\Gamma=q_{0}^{2} / \varepsilon, \Gamma^{\prime}=\Gamma\left(\varepsilon^{\prime}-\varepsilon\right) /\left(\varepsilon^{\prime}+\varepsilon\right)$, and $h=4 \pi q_{0} \sigma /\left(\varepsilon^{\prime}+\varepsilon\right)$ are the coupling parameters governing the strengths of Coulomb interactions among the monomers themselves, between the polymer and its image, and between the polymer and the charged 
surface, respectively. In good solvent regime, $\omega>0$ in the last term.

\section{MONTE-CARLO RESULTS}

The continuum model is discretized to perform MonteCarlo simulations. $\vec{r}(s)$ is replaced by a chain of beads $\vec{r}_{i}$ $(i=1, \ldots, N)$ with hard-core excluded volume of finite radius $a$. Polymer lengths up to $N=120$ are employed. The units of length and energy are $2 a$ and $q_{0}^{2} / 2 \varepsilon a$, respectively. The ratios $\varepsilon^{\prime} / \varepsilon$ are studied from 2 to 12.5 (aqueous solution with a metallic substrate). Runs up to $10^{9} \mathrm{MC}$ steps are performed.

The adsorption behavior can be characterized by normalized monomer density $\rho(z) . \rho_{a} \equiv \rho(z=a)$, the probability density of monomers adsorbed on the substrate, is chosen as the order parameter to describe the adsorption transition. $\rho_{a}$ as a function of the surface charge density $\sigma$ for various $\varepsilon^{\prime} / \varepsilon>1$ is shown in Fig 1 It is seen that $\rho_{a}$ vanishes abruptly when $\sigma$ increases up to its threshold value $\sigma_{\mathrm{t}}$ indicating a first-order transition. At low enough ionic strength that $\kappa^{-1}$ is much larger than absorption layer thickness, $\sigma_{\mathrm{t}}>0$ for $\varepsilon^{\prime} / \varepsilon>1$. The discontinuous drop of $\rho_{a}$ across the transition decreases to zero as $\varepsilon^{\prime} / \varepsilon \rightarrow 1$. We have also verified that the sharp jump in energy (latent heat) across the transition is proportional to $N$ as expected for a first-order transition. Same results are obtained for larger $\kappa^{-1}$.

Furthermore, $\rho_{a}$ is linear in $\sigma$ with the slope depending on $\varepsilon^{\prime} / \varepsilon$. Such a linear relation between $\rho_{a}$ and $\sigma$ can be understood from the electrostatic boundary conditions that the system has to satisfy. The electric potential $\phi(z)$ in the neighborhood of $z=0$ boundary obeys

$$
-\left.\frac{\partial \phi}{\partial z}\right|_{z=0^{+}}+\left.\frac{\partial \phi}{\partial z}\right|_{z=0^{-}}=-\frac{4 \pi}{\varepsilon}\left(\frac{2 \sigma}{\varepsilon^{\prime} / \varepsilon+1}+\sigma_{\mathrm{p}}\right)
$$

where $\sigma_{\mathrm{p}}$ is the polarization surface charge density induced by the polyelectrolyte only. Notice that $\sigma_{\mathrm{p}}$ depends only on $\varepsilon^{\prime} / \varepsilon$. It is independent of $\sigma$ in the adsorbed regime near the transition. The reason is based on the electric blob picture which will be explained in next section. If one treats the polyelectrolyte as a marcomolecule with a well-defined surface, its surface charge density at $z=a$ should be proportional to the monomer density $\rho_{a}$. It also applies to the electric field in the $z<0$ region. One have

$$
\begin{aligned}
& \left.K \frac{\partial \phi}{\partial z}\right|_{z=a^{-}}=-\frac{4 \pi}{\varepsilon} \rho_{a}, \\
& \left.K \frac{\partial \phi}{\partial z}\right|_{z=0^{-}}=-\frac{4 \pi}{\varepsilon} \frac{2 \varepsilon^{\prime}}{\varepsilon^{\prime}+\varepsilon} \rho_{a}
\end{aligned}
$$

where $K>0$ is the proportionality constant. Applying the electric field continuity from $z=0^{+}$to $z=a^{-}$, and

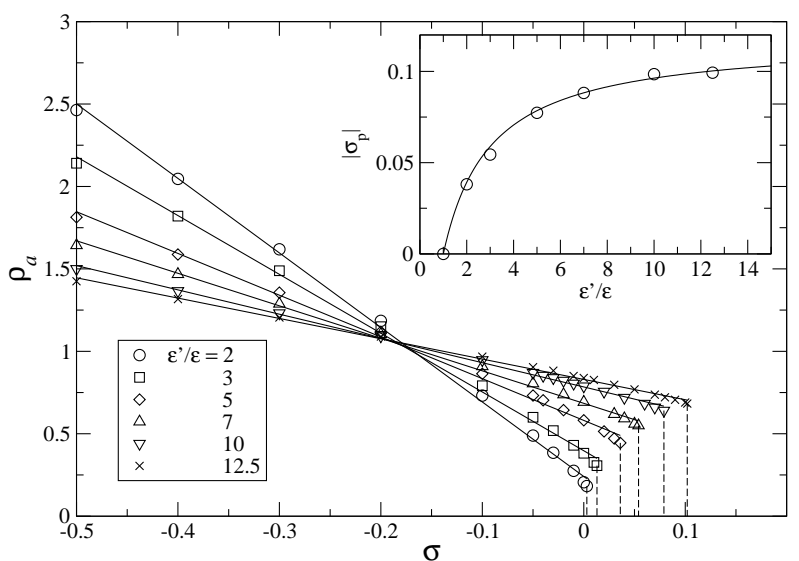

FIGURE 1. Monte-Carlo results for the normalized monomer density at the surface, $\rho_{a}$, as a function of surface charge density $\sigma$ (in unit of $q_{0} / 4 a^{2}$ ) for different $\varepsilon^{\prime} / \varepsilon$ at $\kappa^{-1}=25$. The fitted straight lines are terminated at their adsorption transition points. The vertical dashed lines are drawn as guides to the eyes. Inset: The polarization surface charge density due to the charged polymer, $\left|\sigma_{\mathrm{p}}\right|$, as a function of $\varepsilon^{\prime} / \varepsilon . \sigma_{\mathrm{p}}$ saturates as $\varepsilon^{\prime} / \varepsilon \rightarrow \infty$. The sign of $\sigma_{\mathrm{p}}$ is opposite to $q_{0}$ and is thus negative. The solid curve is fitted from Eq.6. with $\sigma_{\text {poly }}=0.118$.

using Eqs.(2)-(4), one get the linear behavior

$$
\rho_{a}=-\frac{2 K}{\varepsilon^{\prime} / \varepsilon-1}\left(\sigma+\frac{\varepsilon^{\prime} / \varepsilon+1}{2} \sigma_{\mathrm{p}}\right) .
$$

Notice that $K$ and $\sigma_{\mathrm{p}}$ depends only on $\varepsilon^{\prime} / \varepsilon$. As shown in Fig 1 the slope $K$ decreases monotonically with $\varepsilon^{\prime} / \varepsilon$. Lines with different values of $\varepsilon^{\prime} / \varepsilon$ intersect at a common point implies the naive intuition that a more polarizable substrate gives a stronger adsorption is not always true.

Without substrate surface charge, set $\sigma=0$ in Eq. (5), one get

$$
\sigma_{\mathrm{p}}=-\frac{\left.\rho_{a}\right|_{\sigma=0}}{K} \frac{\varepsilon^{\prime} / \varepsilon-1}{\varepsilon^{\prime} / \varepsilon+1}
$$

The dependence on $\varepsilon^{\prime} / \varepsilon$ can be considered as a flat surface of surface charge density $\left.\sigma_{\text {poly }} \equiv \rho_{a}\right|_{\sigma=0} / K$ located above the substrate. $K$ are obtained by linear fittings in Fig 1 and hence $\sigma_{\mathrm{p}}$ as a function of $\varepsilon^{\prime} / \varepsilon$ is plotted in the inset. $\sigma_{\mathrm{p}}$ increases from zero to a saturated value as $\varepsilon^{\prime} / \varepsilon \rightarrow \infty$. Presumably, Eq. (6) suggests that it is possible to experimentally detect the sign and magnitude of the charge of the polyelectrolyte by measuring the substrate polarization.

\section{ANALYTICAL RESULTS}

At low ionic strength, a polyelectrolyte can be treated as electric blobs arranged longitudinally. The rod-like 
polyelectrolyte tends to lie down on the substrate so as to lower its energy. It is thus rigid (rod-like) in $x-y$ plane, but still flexible in the $\mathrm{z}$-direction. In the adsorbed regime, decreasing $\sigma$ (chain is attracted more by the surface) would cause the rearrangement of the electric blobs such that the fluctuations in the z-direction is reduced (and hence the layer thickness decreases), but the fluctuations in the other two directions and the size of the blobs are basically unchanged. The effective in-plane surface charge distribution of the polyelectrolyte, and hence $\sigma_{\mathrm{p}}$, is not affected by $\sigma$. The excluded volume effect is ignored because it takes almost no effect in z-direction. The effect from self-electrostatic interaction can be absorbed by renormalising the bare persistence length $l_{0}$ to $l$.

Because the monomer would feel the strongest attraction from its direct image around the adsorption regime, the $\Gamma^{\prime}$-term in Eq. (1) is approximated by the interaction of every monomer and its corresponding image only. The residual attraction from the images of other monomers could be absorbed into the coupling parameter $\Gamma^{\prime}$ by renormalising $q_{0}$ to $q$. The partition function is reduced to

$$
\begin{aligned}
Z= & \int \mathscr{D}[\vec{r}(s)] \exp \left[\int _ { 0 } ^ { N } d s \left\{-\frac{3}{2 l^{2}}\left(\frac{\partial \vec{r}(s)}{\partial s}\right)^{2}\right.\right. \\
& \left.\left.+\frac{\beta \Gamma^{\prime}}{4} \frac{\mathrm{e}^{-2 \kappa \vec{r}(s) \cdot \hat{z}}}{\vec{r}(s) \cdot \hat{z}}-\beta h \kappa^{-1} \mathrm{e}^{-\kappa \vec{r}(s) \cdot \hat{z}}\right\}\right] .
\end{aligned}
$$

$\vec{r}(s)$ is transformed to $\rho(\vec{r}) \equiv \frac{1}{N} \int_{0}^{N} d s \delta(\vec{r}-\vec{r}(s))$ by introducing an auxiliary field. Ground state dominance in large- $N$ limit is then applied. By variational principle, one obtains the Edwards-Schrödinger equation,

$$
\left(-\frac{l^{2}}{6} \frac{d^{2}}{d z^{2}}-\frac{\beta \Gamma^{\prime}}{4} \frac{\mathrm{e}^{-2 \kappa z}}{z}+\beta h \kappa^{-1} \mathrm{e}^{-\kappa z}\right) \psi(z)=\varepsilon_{0} \psi(z)
$$

where $\varepsilon_{0}$ acts as a Lagrange multiplier to enforce the constraint of the ground state wavefunction normalization. The monomer density is given by $\rho(z)=|\psi(z)|^{2}$. Eq. (8) also describes a quantum particle at its ground state moving under a combined potential of a $1 \mathrm{~d}$ screened Coulomb attraction and an almost linear potential. However, the boundary condition expressed by Eq. (5) is different from that of the hard-wall one $\left.\psi\right|_{\mathrm{s}}=0$ usually employed for the quantum particle. Instead $\left.\psi\right|_{\mathrm{s}}=\sqrt{\rho_{a}} \neq 0$ for the present problem implies that the steric force felt by the polyelectrolyte from the charged surface should be modified [8]. Setting $\left.\psi\right|_{\mathrm{s}}=0[6,7,9]$ in previous studies is not completely correct.

During the adsorption, the rod-like polyelectrolyte tends to lie down on the charged surface. The thickness of the adsorption layer is of the same order of the gyration radius in $\mathrm{z}$-direction. At low ionic strength corresponding to Debye length much larger than layer thick-

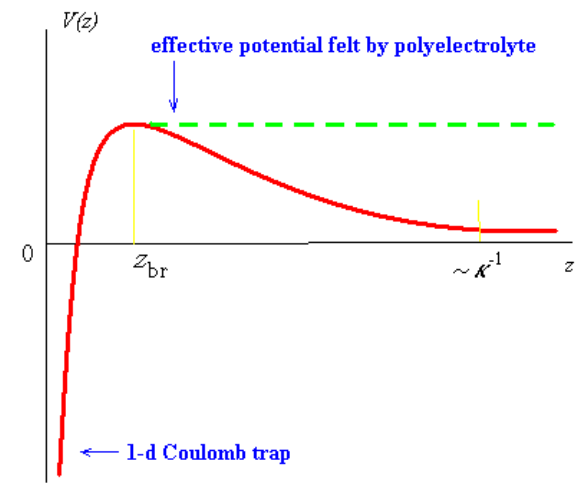

FIGURE 2. Effective Potential felt by the polyelectrolyte

ness, the polyelectrolyte can only feel the potential barrier formed by $V(z)$ as shown in Fid2 Analytically, the original potential $V(z)$ in Eq. 8 is replaced by

$$
V_{\text {mod }}(z)= \begin{cases}+\infty, & z<a \\ V(z), & a \leq z<z_{\mathrm{br}} \\ V\left(z_{\mathrm{br}}\right), & z \geq z_{\mathrm{br}}\end{cases}
$$

where $z_{\mathrm{br}}$ is chosen such that $V^{\prime}\left(z_{\mathrm{br}}\right)=0$, and $V\left(z_{\mathrm{br}}\right)$ is the barrier height. In the limit of $\sigma=\kappa=0$, the analytic solution exists in the form

$$
\psi(z)=\mathrm{W}_{\lambda, 1 / 2}\left(\frac{3 \beta \Gamma^{\prime}}{2 l^{2} \lambda} z\right) ; \varepsilon_{0}=-\frac{3 \beta^{2} \Gamma^{\prime 2}}{32 l^{2}} \frac{1}{\lambda^{2}}
$$

where $\mathrm{W}_{\lambda, 1 / 2}$ is the Whittaker's notation of the confluent hypergeometric function [10], and $\lambda$ is the least value satisfying the boundary condition. Bound state exists for arbitrary $\varepsilon^{\prime} / \varepsilon>1$. It implies $\sigma_{\mathrm{t}}>0$ at low enough ionic strength.

No exact solution exists for $\sigma, \kappa>0$ in general but one can analyze it around the transition. In the region of interest, the Coulomb term dominates over the almost linear term. One can approximate the binding energy by Eq. 10]. The polyelectrolyte undergoes a de-sorption transition when the binding energy is equal to barrier height $V\left(z_{\text {br }}\right)$. After some algebra, it shows $\sigma_{\mathrm{t}} \sim\left(\varepsilon^{\prime} / \varepsilon-\right.$ 1) for $\varepsilon^{\prime} / \varepsilon \gg 1$ and $\sigma_{\mathrm{t}} \sim\left(\varepsilon^{\prime} / \varepsilon-1\right)^{3}$ for $\varepsilon^{\prime} / \varepsilon \sim 1$. This analytic result is consistent with simulation as shown in Fig 3.

An approximate solution for density profile $\rho(z)$ for $\sigma>0$ can be studied by the variational wavefunction

$$
\psi(z)=\sqrt{\rho_{a}}(1+\mu \alpha(z-a)) \mathrm{e}^{-\frac{1}{2} \alpha(z-a)}
$$

where $\alpha^{-1}$ is the decay length. $\mu$ is positive because the wavefunction is restricted to be nodeless. $\alpha$ and $\mu$ are not independent but related via the wavefunction normalization condition. The inverse decay length is calculated to 

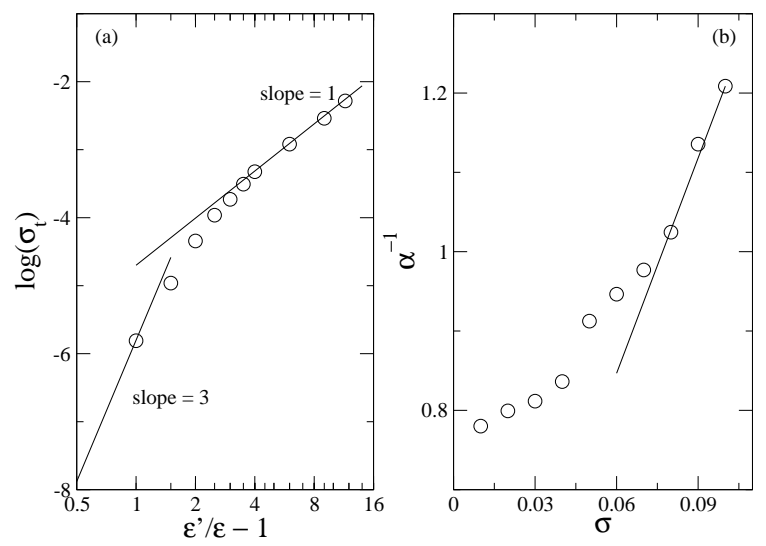

FIGURE 3. (a) Simulation results for the surface charge density at the transition, $\sigma_{\mathrm{t}}$, as a function of $\varepsilon^{\prime} / \varepsilon$ in logarithmic scale at $\kappa^{-1}=25$. The straight lines indicate slopes of 1 and 3 as suggested in the text. (b) Simulation results for the decay length $\alpha^{-1}$ (which is proportional to the layer thickness) as a function of $\sigma$ (in unit of $q_{0} / 4 a^{2}$ ) for $\varepsilon^{\prime} / \varepsilon=12.5, \kappa^{-1}=25$. The straight line is a linear fit to points near the transition. $\sigma_{\mathrm{t}}=0.102$ in this case. $\alpha^{-1}$ is obtained from exponential fitting to the tail of corresponding density profile.

be

$$
\alpha=\frac{3 \beta \Gamma^{\prime}}{2 l^{2}}+\rho_{a}
$$

where the leading term is independent of $\sigma$. Near the transition, the decay length $\alpha^{-1}$ and hence the adsorption layer thickness $D \sim \alpha^{-1}$ remains finite. Combining Eqs.(5) and (12), one get the scaling behavior

$$
D_{\mathrm{t}}-D \sim\left(\alpha_{\mathrm{t}}^{-1}-\alpha^{-1}\right) \sim\left(\sigma_{\mathrm{t}}-\sigma\right)
$$

where $D_{\mathrm{t}}$ is the threshold layer thickness. $\alpha^{-1}$ as a function of $\sigma$ from simulations is shown in Fig 3 b.

It would be beneficial to compare with the case that a polyelectrolyte is adsorbed onto an attractive charged substrate of low-dielectric constant (e.g. DNA in aqueous solution onto a charged lipid membrane). Its asymptotic solution for $z \rightarrow+\infty$ to Eq. (8) reproduces the usual scaling $D \sim \alpha^{-1} \sim|\sigma|^{-\frac{1}{3}}[4]$. The adsorption onto a lowdielectric substrate is continuous. The order parameter $\rho_{a}$ vanishes continuously across the transition, and the layer thickness swells to infinity as the polyelectrolyte is desorbed [11].

\section{DISCUSSION}

A strongly charged polyelectrolyte immersed in a salt solution will attract oppositely charged ions to condense until its effective charge density reaches the Manning threshold [12]. This means that one can just renormalize $q_{0}$ in our system to $2 e a / l_{\mathrm{B}}$ if $q_{0}$ is larger than $2 e a / l_{\mathrm{B}}\left(l_{B}\right.$ is the Bjerrum length). Similarly, the strongly charged surface of bare charge density larger than $\kappa /\left(\pi l_{\mathrm{B}}\right)$ is just renormalized back to $\kappa /\left(\pi l_{\mathrm{B}}\right)$ [13].

Although we focus on the adsorption of a single polyelectrolyte, our results may provide a starting point to study the charge inversion and multi-layer adsorption. [14]. At low ionic strength, polyelectrolytes are adsorbed in a multi-layer structure because of strong Coulomb repulsion. Each layer is composed of the parallel 1d Wigner crystal [15]. From our physical picture of a single polyelectrolyte adsorption, the upper bound for the thickness of the multi-layer structure is $z_{\mathrm{br}} \sim \sigma^{-1 / 2}\left(\varepsilon^{\prime} / \varepsilon-\right.$ $1)^{1 / 2}$. This suggests one can easily control just one single layer adsorbed onto high-dielectric substrate by tuning the surface charge density. Rigorous treatment based on this physical picture will be elaborated elsewhere.

\section{REFERENCES}

1. de Gennes, P.G., Scaling Concepts in Polymer Physics, Cornell Univ., New York, 1979, pp.252-254.

2. Gelbert, W.M., Bruinsma, R.F., Pincus, P.A., and Parsegian, V.A., Physics Today 53, 38, (2000).

3. Grosberg, A.Y., Nguyen, T.T., and Shklovskii, B.I., Rev. Mod. Phys. 74, 329 (2002).

4. Joanny, J.F., Eur. Phys. J. B9, 117 (1999).

5. Nguyen, T.T., Grosberg, A.Y., and Shklovskii, B.I., Phys. Rev. Lett. 85,1568 (2000).

6. Wiegel, F.W., Introduction to Path-Integral Methods in Physics and Polymer Science, World Sci., Singapore, 1986, pp.39-40.

7. Muthukumar, M., J. Chem. Phys. 86, 7230 (1987).

8. Cheng, C.H. and Lai, P.Y. (unpublished).

9. Netz, R.R. and Andelman, D., Phys. Rep. 380, 1 (2003).

10. Loudon, R., Am. J. Phys. 27, 649 (1959).

11. Cheng, C.H. and Lai, P.Y. (unpublished).

12. Manning, G.S., J. Chem. Phys. 51, 924 (1969).

13. Bocquet, L., Trizac, E., and Aubouy, M., J. Chem. Phys. 117, 8138 (2002).

14. Dobrynin, A.V., Deshkovski, A., and Rubinstein, M., Macromolecules 34, 3421 (2001).

15. Shklovskii, B.I., Phys. Rev. Lett. 82, 3268 (1999). 